\title{
Léxico funerario en el aula ELE: colocaciones léxicas con el verbo celebrar y léxico afín
}

\author{
LAURA ARROYO MARTÍNEZ \\ Universidad Rey Juan Carlos \\ laura.arroyom@urjc.es
}

\section{Resumen}

El presente artículo tiene como finalidad demostrar el interés que posee incluir el léxico funerario en la clase de ELE para mejorar la competencia léxica y sociocultural de nuestros estudiantes. Es necesario resaltar su interés puesto que este léxico es un gran olvidado en las programaciones. De mismo modo, presenta una explicación didáctica, así como una propuesta de actividades, basada de la lingüística cognitiva y en el enfoque léxico que permite demostrar cómo se puede enseñar este léxico con una metodología eficiente.

\section{Palabras clave}

Léxico funerario; competencia sociocultural; enfoque léxico; propuesta actividades. 


\title{
Funeral lexicon in SFL's classroom: lexical collocations with the verb celebrate and related lexicon
}

\begin{abstract}
The purpose of this article is to demonstrate the interest of including the funeral lexicon in the ELE class to improve the lexical and sociocultural competence of our students. It is necessary to highlight your interest since this lexicon is widely forgotten in programming. In the same way, it presents a didactic explanation, as well as a proposal of activities, based on cognitive linguistics and on the lexical approach that allows to demonstrate how this lexicon can be taught with an efficient methodology.
\end{abstract}

\section{Keywords}

Funeral lexicon; sociocultural competence; lexical approach; activities proposal.

Recibido el 21/05/2020

Aceptado el 09/11/2020 


\section{Introducción: el silencio sepulcral sobre el léxico funerario en las programaciones de ELE}

Una de las preguntas que todo profesor de ELE se plantea al abordar los contenidos que va a incluir en la programación de su curso es qué léxico va a enseñar. Resolver esta cuestión no es una tarea sencilla porque es evidente que nuestros alumnos no pueden incorporar a su lexicón mental todas las palabras de la lengua extranjera o segunda lengua que están aprendiendo. Por esta razón, entre otras, los criterios de selección son muy complejos y, como ya señalaba en 2008 Gómez Molina, la elección de unos campos semánticos o de unas áreas temáticas frente a otras se ve condicionada por el contexto comunicativo y cultural en el que se insertan los mismos:

No hay que olvidar que estos temas de discurso, materia de conversación, reflexión y composición, se inscriben en el contexto de una situación comunicación específica dentro de los diferentes ámbitos en que se organiza la vida social y, a su vez, las situaciones comunicativas que surgen en cada uno de ellos puede referirse a lugares y momentos, instituciones, personas implicadas, objetos del entorno, acontecimientos que suceden, intervenciones realizadas por las personas implicadas y los textos (géneros discursivos) empleados en dicha situación. (Gómez Molina, 2008: 796)

Por consiguiente, no todos los campos léxicos se trabajan al mismo nivel en las aulas de lenguas extranjeras. Resulta reseñable que el campo léxico relacionado con la muerte y sus ritos apenas se aborda ni en las programaciones didácticas, ni en los materiales de ELE de las diversas editoriales especializadas (no he encontrado ningún manual que lo incluya). Sin embargo, este silencio pedagógico choca con la exposición de contenidos léxicos sobre este tema que delimita con absoluta claridad el PCIC ${ }^{1}$. Indico, a continuación, qué elementos se incluyen de manera explícita dentro del repertorio léxico descrito en el citado texto.

Además del verbo morir que los alumnos deben emplear desde el nivel A, en niveles superiores el alumnado debe incrementar su capacidad léxica en este campo semántico. En el nivel B2, el alumno debería saber utilizar verbos como enterrar, incinerar y fallecer. En el nivel C1 deben conocer los sustantivos entierro, incineración, difunto, fallecido y expresiones fraseológicas como «estirar la pata». En el nivel C2 debe manejar términos como restos mortales, defunción, óbito, deceso, sepelio, inhumación, expirar, sepultar, inhumar y momificar; así como otros más concretos sobre los lugares y objetos asociados a los ritos funerarios: nicho, ataúd, lápida, sepulcro, sepultura, fosa o sarcófago. Por último, también se deben emplear modismos asociados al tema como «irse al otro barrio», «pasar a mejor vida» y «criar malvas», lo que indica que los estudiantes deben diferenciar con nitidez los registros lingüísticos asociados a diversas situaciones comunicativas.

1 Véase https://cvc.cervantes.es/ensenanza/biblioteca_ele/plan_curricular/ 
Por tanto, el PCIC señala claramente que el aprendiente debe conocer diferentes palabras asociadas al tema de la muerte y, sin embargo, esto no se refleja en el aula de ELE. La pregunta que inmediatamente surge es por qué ocurre esto. Es importante resolver esta cuestión para saber si nos encontramos en la buena dirección al no prestar atención a este léxico dentro del aula o, por el contrario, debemos empezar a incluirlo en nuestras programaciones. Algunos argumentos que considero que justifican este olvido léxico son los que se presentan a continuación.

En primer lugar, considero que hay una predilección por abordar áreas temáticas que se incluyen dentro de las pruebas de evaluación de los exámenes oficiales. Así, el Instituto Cervantes explicita cuáles son las áreas temáticas que se integran dentro de sus exámenes oficiales y que citamos a continuación: identificación personal, casa y alojamiento, trabajo, estudios, y ocupación, tiempo libre, viajes y transportes, relaciones sociales, salud y estado físico, compras, comidas y bebidas, edificios de servicios públicos, tiempo y clima y, por último, problemas de comunicación. Si analizamos con detenimiento este listado, la experiencia como profesores de ELE nos hace saber que, efectivamente, encontramos estas áreas temáticas de manera reiterativa (hasta de manera abusiva) en programaciones y manuales. Este hecho no es fruto de la casualidad, porque toda institución de enseñanza, así como todo docente, es consciente de la importancia de estas pruebas de certificación oficial para un amplio conjunto de nuestros estudiantes. Por tanto, se puede deducir que estas áreas temáticas no se trabajan de manera redundante sólo por su alto rendimiento comunicativo (que lo tienen, indiscutiblemente), sino por un enfoque orientado claramente a la evaluación de procesos futuros. No podemos obviar que el peso de la evaluación dentro de todo el proceso formativo de los alumnos sigue teniendo una repercusión muy destacada en la organización de los programas dentro de los diversos sistemas educativos de carácter formal.

En segundo lugar, en la gran mayoría de las culturas existe un tabú instaurado sobre el tema de la muerte; situación que se traslada al aula de ELE. Pese al auge indiscutible que en los últimos años se ha otorgado a la competencia cultural, como competencia esencial que deben alcanzar los alumnos que cursan diversos programas de lenguas extranjeras y segundas lenguas, el tema de la muerte y sus ritos no ha sido incorporado, pese a poseer un alto valor cultural. Sin duda alguna esto tiene que interpretarse a la luz del interrogante de qué cultura se enseña en nuestras aulas. Una de las principales vías de explotación de contenidos culturales está marcada por un claro enfoque comparativo. Por ejemplo, comparamos las fiestas tradicionales de los países de habla hispana con las de los países de origen de nuestros estudiantes; del mismo modo procedemos con las celebraciones familiares y sociales, la gastronomía, las viviendas, la música, el cine, las artes, y tantos otros temas. Si analizamos los ejemplos que he presentado anteriormente, se puede deducir que todos comparten dos características comunes: ninguno de ellos provoca conflictos ideológicos que dificultan la gestión del aula y, además, a nivel emocional (aspecto esencial como elemento motivacional) todas estas áreas temáticas se asocian a sensaciones agradables, placenteras, es decir, permiten al alumno rememorar momentos felices de su propia biografía. 
Si partimos de las dos consideraciones anteriores, nuestro razonamiento nos conduce al tercer inconveniente: nos encontramos con el difícil manejo ideológico que puede presentar este tema. Lo primero que tenemos que pensar es que la muerte es un concepto desde el punto de vista antropológico, claramente ligado a la religión, aspecto especialmente delicado por pertenecer al ámbito íntimo de los alumnos. Pensemos que en todos los países en los que el español es lengua oficial o cooficial, la religión mayoritaria es el cristianismo. Esta religión ha dejado una impronta esencial a lo largo de los siglos en la cultura y las tradiciones de estos países. Por tanto, la concepción de la muerte y de las ceremonias religiosas y sociales que la rodean está íntimamente ligada con el cristianismo, del que es inseparable. Pongamos por ejemplo la celebración de una misa funeral en memoria de una persona. Este acto religioso solo se puede entender desde una visión cristiana. Por tanto, imaginemos una clase de español para extranjeros en la que tengamos alumnos musulmanes, budistas, hinduistas, o practicantes de otras religiones; así como alumnos ateos o agnósticos. No es complicado afirmar que sus creencias religiosas obligan a asumir de manera diferencial la muerte, con todas sus implicaciones trascendentales. Por tanto, este tema se tendría que abordar con una planificación previa muy controlada (actividades cerradas), se tendría que focalizar su interés sobre el léxico y la cultura social sobre la muerte, pero no entrar en ningún caso en discusiones religiosas de calado y, además, se tendría que realizar un estudio previo sobre la composición de nuestra aula. Algunas preguntas previas que el docente debe plantearse serían las siguientes: ¿Todos nuestros alumnos poseen la misma religión? ¿Tengo alumnos de diversas religiones en el aula? ¿Cómo asumen su religión? ¿Es un tema de especial relevancia para ellos? No es un aspecto que podamos pasar por alto. Por tanto, el tema de la muerte se debe tratar en el aula de lenguas extranjeras, pero siempre se debe abordar con la sensibilidad escrupulosa que merece.

En cuarto y último lugar, cabe mencionar la importancia de la emotividad dentro del aula de lengua extranjera. Está comprobado que

el factor afectivo ayuda a la retención de la lengua meta y permite recuperarla cuando la situación comunicativa lo requiera. Es obvio, que lo que nos suscita curiosidad o nos motiva lo recordamos mejor que aquello que nos aburre. [...] Más aún, si las formas lingüísticas van unidas a componentes afectivos intensos, éstas se retienen durante más tiempo y se hacen más accesibles para su uso, porque se almacenan en lo que Stevick (2002) llama la memoria permanente. (Fernández-Corbacho y Toscano-Fuentes, 2015, 10)

En relación con este aspecto, como generalización se puede afirmar que en todas las culturas (con diferencias de grado entre ellas), el tema de la muerte posee connotaciones negativas. Muchos hablantes asocian la muerte a sentimientos como la incertidumbre, el miedo, la enfermedad, la pérdida, el duelo; otros, los más religiosos a valores trascendentes. Se considera, por tanto, que abordar el tema de la muerte puede producir en nuestros estudiantes sensaciones desagradables que dificulten su aprendizaje y, por consiguiente, se descarta con demasiada prontitud, como se explicará en el siguiente epígrafe del presente trabajo. 


\section{2. ¿ंPor qué trabajar sobre el área temática de la muerte y sus ritos en el aula de $E / L E$ ?}

En la introducción del presente artículo se han presentado los principales argumentos por los cuales se ha descartado de manera sistemática incluir el área temática de la muerte y sus correspondientes ritos en el aula de ELE o EL2. A continuación, voy a presentar de manera muy sintética una contrargumentación que justifica la necesidad de modificar esta renuncia voluntaria dentro de nuestras programaciones.

Desde el punto de vista de la competencia sociocultural dar a conocer qué eventos sociales se realizan en España u otros países cuando fallece alguien (asistir a un tanatorio para acompañar a familiares o amigos, asistir a un entierro o a una misa funeral, acudir al cementerio para honrar a nuestros difuntos etc.) y cómo nos comportamos y nos expresamos en dichas circunstancias sociales resulta de gran interés. Si trabajamos para conseguir alcanzar objetivos lingüísticos y comunicativos respecto a eventos como bodas, cumpleaños y celebraciones de todo tipo, así como otro tipo de situaciones de la vida cotidiana, porque consideramos que puede ser necesario para que nuestros alumnos puedan desenvolverse en este tipo de situaciones reales, ¿qué nos lleva a concluir que nuestros alumnos en una situación de inmersión no van a necesitar saber dar el pésame a un amigo o saber cumplir con las normas socialmente esperables en la visita a un tanatorio? No hay ningún tipo de argumento que permita razonar de esta manera, máxime cuando estas circunstancias se rodean de actos de habla que ya resultan costosos o incómodos para hablantes nativos, que incluso pueden mostrar sus propias limitaciones comunicativas. A esto, sumaríamos el interés que tiene el conocimiento de fiestas como el Día de Difuntos, que en países como España o México resulta fundamental.

Este razonamiento se refuerza al recordar que es el propio PCIC el que en el apartado dedicado al inventario de «Saberes y comportamientos socioculturales», y dentro de éste, en el apartado «Identidad colectiva y estilo de vida», explicita los temas que se deben abordar en los siguientes términos:

se incluyen todos los aspectos que permiten a los individuos sentirse parte integrante de una sociedad. Tiene que ver, pues, con las convenciones sociales respecto a los saludos, las despedidas, el comportamiento en celebraciones, fiestas, invitaciones $\mathrm{o}$ en determinados actos sociales, como bodas $\mathrm{o}$ cumpleaños; con el comportamiento ritual colectivo en las ceremonias y las prácticas religiosas, el nacimiento o la muerte; con la participación ciudadana en la esfera social y con las tradiciones que perviven y las que son cuestionadas por nuevos estilos de vida y de relaciones ${ }^{2}$.

Las dificultades ideológicas aludidas en la introducción de este trabajo pueden convertirse en una fuente de enriquecimiento cultural. Es decir, la clase de ELE no tiene

${ }^{2}$ https://cvc.cervantes.es/ensenanza/biblioteca_ele/plan_curricular/niveles/11_saberes_y_comportamientos_ introduccion.htm 
como propósito confrontar ideologías o, en este caso, religiones, sino todo lo contrario: debe convertirse en un escenario multicultural y abierto, que fomente el respeto entre culturas. Desde este prisma, el tema de la muerte puede ser tratado con una perspectiva antropológica y cultural que pueda interesar a aquellos alumnos que tengan un perfil intelectual marcado en su lengua materna. Este tipo de alumnado puede tener interés en conocer los ritos y costumbres funerarias de otras culturas con el enfoque anteriormente citado. Para ellos, la clase de ELE permitiría ampliar su conocimiento del mundo más allá de su conocimiento lingüístico.

En relación con la importancia de la gestión de emociones en la adquisición de lenguas extranjeras, como se ha explicado anteriormente, crear emociones es esencial para que el alumno recuerde y sepa poner en uso con mucha más facilidad diversos componentes gramaticales y, principalmente, léxicos. Se pretende trasladar al aula emociones gratificantes y/o divertidas para el alumno. En primer lugar, se piensa que una emoción positiva se recuerda mejor que una negativa. Sin embargo, ambas se rememoran con intensidades similares. Si nos hacemos una pregunta tan sencilla como la siguiente: ¿recordamos mucho más la boda de un familiar cercano que el fallecimiento de otro familiar cercano? La respuesta es que el recuerdo de estos dos eventos clave en nuestra trayectoria personal puede poseer intensidades similares, siendo uno festivo y otro, doloroso. Como justifica Méndez López, el tratamiento de las emociones, tanto positivas como negativas, constituye un parámetro motivador dentro del aula de lenguas extranjeras:

Emotions, whether negative or positive, have both a negative or positive influence. [...] Although no clear conclusions have been established regarding the shaping effects of intensity and frecuency of emotions in learning, negative or positive emotions have been found to enhance not only learning processes but also the development of learners. (Méndez López, 2016: 28)

Además del grado de intensidad similar que se ha referido, la orientación del profesor desde el punto de vista de la emoción puede constituir la clave del éxito. En los comportamientos sociales asociados a la muerte encontramos comportamientos de familiares y amigos que nos reconfortan y que también dejan una gran huella en nuestros recuerdos: experiencias vitales que se pueden llevar al aula de ELE.

Por último, el efecto sorpresa en el aula puede ser otra razón por la que incluir esta área temática en nuestras programaciones. Dentro de cualquier proceso de aprendizaje es necesario sacar al alumno de su zona de confort y romper en algunas ocasiones su monotonía. El alumno está acostumbrado (espera) que su profesor de español le proponga organizar y preparar un viaje con sus compañeros de clase, ver y comentar fragmentos de una película en español o redactar una carta de motivación para postularse como candidato a un puesto de trabajo. Sin embargo, se sorprendería notablemente si le pidiéramos realizar una llamada para consolar a un familiar al que se le ha muerto su padre, escribir un e-mail para dar el pésame a un compañero de trabajo o preparar una exposición oral para presentar a sus compañeros de clase cómo celebramos el Día de Difuntos en España u otros países y qué fiesta comparable existe 
en su cultura. Trabajar con esta área temática nos permitiría, tal vez, recuperar parte de la atención perdida en el desgaste de un curso estándar.

Por estas razones, entre otras, consideramos que existen ventajas notables para empezar a incluir estos contenidos y, por consiguiente, el léxico relacionado con los mismos, en las programaciones de nuestros cursos, principalmente en los niveles intermedio y superior. Por esta razón, en los siguientes epígrafes se va a desarrollar el enfoque lingüístico que considero más oportuno para llevarlo a cabo para, posteriormente, presentar un ejemplo de combinación léxica con el verbo celebrar que permita servir de modelo a una propuesta de actividades.

\section{Las combinaciones léxicas con el verbo celebrar mediante una explicación cognitiva y con un enfoque léxico dentro del aula ELE}

Este epígrafe presenta como objetivo principal analizar las posibles colocaciones léxicas válidas que se forman con el verbo celebrar acompañado de sustantivos relacionados con la muerte. Es decir, dentro de la tipología de colocaciones clásica realizada por Corpas (1996: 66-76), que tiene presente la categoría gramatical y la relación sintáctica que se da entre los elementos de la colocación, las colocaciones que se estudian en este artículo pertenecen a las de tipo verbo + sustantivo ${ }^{3}$.

Desde mediados del siglo pasado cuando Firth designó el concepto por primera vez como collocation hasta nuestros días, disponemos de una amplia bibliografía que ha mantenido un profundo debate sobre qué es una colocación, las cualidades que estas unidades léxicas poseen, así como su diversidad tipológica ${ }^{4}$. Desde un enfoque estrictamente didáctico en la enseñanza de segundas lenguas del que parte este artículo, las colocaciones son «un tipo de unidades léxicas, que el profesor tiene que resaltar en el input ${ }^{5}$ para que el alumno aprenda las combinaciones frecuentes de las palabras en una lengua extranjera y que se facilite la creación de redes de significados que permitan memorizarlas en el lexicón» (Higueras García, 2006, 18)

Uno de los centros de interés en la investigación sobre la adquisición del léxico se encuentra en comprender los mecanismos mediante los cuales nuestros alumnos son capaces de memorizar ${ }^{6}$ e integrar el caudal léxico que necesitan para comunicarse en

\footnotetext{
${ }^{3}$ Dentro de este grupo se encuentran tres subgrupos: las colocaciones que comparten el colocativo y una base perteneciente al mismo campo; las colocaciones cuyas bases presentan un patrón de combinación limitado y las colocaciones intermedias en las que se incluyen verbos deslexicalizados.

${ }^{4}$ No es el objetivo de este artículo revisar dicho recorrido teórico. Para el lector que necesite recuperar esta información recomendamos el capítulo «El concepto de colocación» que se encuentra en el manual Las colocaciones y su enseñanza en la clase de ELE, de Marta Higueras García (2006), cuya referencia completa se encuentra en la bibliografía.

${ }^{5}$ No es objetivo de este trabajo analizar si el léxico se debe adquirir únicamente de manera indirecta, a través del contacto con la lengua. El debate sobre esto ha sido muy profundo entre los expertos en adquisición de lenguas extranjeras. Simplemente quiero apuntar que considero que el léxico también se debe trabajar de manera explícita, mediante la propuesta de actividades que lo presenten de manera contextualizada.

${ }^{6}$ Afortunadamente, en la actualidad hay un acuerdo unánime en que, frente a lo que ocurría en la metodología estructuralista, en ningún caso se debe «abrumar al estudiante con las tradicionales listas de palabras para que las aprenda más o menos memorísticamente. [...] parece ampliamente demostrado que este procedimiento de enseñanza no es el más adecuado, sobre todo en la adquisición de segundas lenguas» (Arribas Esteras, 2004, 931).
} 
una segunda lengua. Este proceso es muy complejo porque hoy ningún investigador niega que «saber una palabra es conocer, por un lado, su forma y el significado, y, por otro lado, saber usarla» (Rufat, 2016: 172) Es decir, en el contexto de las nuevas metodologías se asume que todo el léxico debe trabajarse dentro del aula con especial atención al contexto en el que las palabras se emplean, por lo que la competencia léxica se relaciona íntimamente con la competencia pragmática y sociocultural7. Partiendo de estos principios, la construcción del lexicón que realiza cada uno de nuestros estudiantes a lo largo de su proceso de aprendizaje es única y verdaderamente compleja. Como sintetiza Higueras García:

Los recientes estudios en psicolingüística permiten afirmar que la adquisición del léxico no es solo una suma de palabras que el alumno va memorizando y almacenando paulatinamente, sino que se trata de un proceso cualitativo, gradual, multidimensional, procesual y dinámico, en el que el aprendiente teje una intrincada red de relaciones -fonéticas, gráficas, semánticas, morfológicas, sintácticas, enciclopédicas o personales- entre unidades léxicas, que asocian y fijan la información nueva a la conocida. (Higueras García, 2006: 13)

Para alcanzar esta meta nos vamos a apoyar en un enfoque basado en las propuestas que ha planteado la lingüística cognitiva, así como en el enfoque léxico ${ }^{8}$, puesto que como se ha indicado con gran acierto, es en la mezcla de ambos enfoques donde se obtiene el rendimiento pedagógico ${ }^{9}$. Por esto, «en los últimos años se ha desarrollado una corriente para promover la retención de las secuencias formulaicas echando mano de la semántica cognitiva» (Pérez Serrano, 2017: 43) Del mismo modo, no nos olvidamos de la importancia del enfoque pragmático, que en la enseñanza del léxico no se ha quedado atrás. Como ha señalado con gran acierto Fernández Montoro, la inclusión de la Pragmática en la didáctica del léxico

vincula el estudio del léxico a una realidad social e histórica, a una realidad cultural. Esta disciplina ayudará a aislar, identificar e interpretar esos otros contenidos que intervienen en un intercambio comunicativo, además aportará orientaciones que pueden dar respuesta a preguntas de adecuación social o cultural al código. (Fernández Montoro, 2014: 7)

\footnotetext{
7 Según Penadés Martínez, para que los alumnos aprendan fraseología habría que tener trabajar también el comportamiento sintagmático de estas construcciones léxicas, algo con lo que estamos plenamente conformes. Como indica, «por lo que se refiere a las actividades enfocadas a su didáctica se ha señalado la oportunidad de proponer ejercicios sobre la adquisición de un vocabulario de la lengua meta, con la finalidad de potenciar la información colocacional sintáctica de una palabra. Lo cual trae aparejada la necesidad de que el alumno conozca el ámbito colocacional de las palabras y se interese por su entorno habitual» (Penadés Martínez, 2004: 59) ${ }^{8}$ No es motivo del presente artículo hacer una revisión histórica de la evolución de la didáctica del léxico en el aula de lenguas extranjeras. Para comprender este recorrido desde el estructuralismo imperante hasta la segunda mitad del siglo XX hasta las teorías lingüísticas más actuales recomendamos en brillante trabajo sintético realizado por Sánchez Rufat titulado «Apuntes sobre la revalorización del léxico en la teoría lingüística y en la enseñanza de lenguas» (2016).

9 Como defiende Pérez Serrano, «hay evidencia empírica de que, si las tareas que proponemos al estudiante llevan aparejadas una implicación cognitiva por parte del mismo y un procesamiento semántico, el aprendizaje es mayor y más significativo» (Pérez Serrano, 2017: 43).
} 
Dentro de la lingüística cognitiva considero interesante rescatar el concepto de categorización para ponerlo al servicio de la adquisición del léxico. Tanto los hablantes nativos como los estudiantes de lenguas extranjeras organizamos el léxico mediante diversos procesos cognitivos ${ }^{10}$. Estos procesos deben ser especialmente potenciados en el aula de ELE para favorecer el aprendizaje de nuestros estudiantes. Uno de los más importantes, sin lugar a duda, es el de la categorización de las palabras, puesto que este proceso nos permite asociar el léxico disponible para mejorar nuestra capacidad de respuesta lingüística (tanto en el plano receptivo, como productivo). De este modo, como defiende la teoría de prototipos, la categorización es un proceso neuropsicológico muy potente para organizar nuestro lexicón mental. Por consiguiente, creamos categorías «mediante la inserción de una o varias propiedades típicas que tienden a coincidir, aunque dicha coincidencia no sea estrictamente necesaria» (Cuenca y Hilferty, 1999: 39)

De esta manera, si atendemos a este enfoque es interesante presentar de forma explícita información sobre la categorización del léxico que queremos que adquieran nuestros estudiantes. En este caso, se presentará el verbo celebrar junto a la combinación posible de sustantivos que pueden funcionar como argumentos, de manera categorizada, asociados por sus elementos prototípicos. Con este tratamiento del léxico, los estudiantes, de manera sistemática y simplificada, podrán reducir sustancialmente su incertidumbre a nivel gramatical y mejorarán su alcance léxico. Como hemos explicado anteriormente, es incontestable que la gramática y el léxico no se procesa de manera aislada, sino que, muy al contrario, el adecuado conocimiento léxico favorece la interiorización de estructuras sintácticas y morfológicas de las lenguas.

Dentro del enfoque léxico, resulta esencia presentar las explicaciones dentro del aula mediante partiendo de la Teoría del lexicón generativo que postula que

el significado de las palabras se construye a través de la interacción entre su información externa (estructura subléxica codificada en forma de rasgos semánticos) y el contexto. Es decir, el contexto influye en la construcción del significado de las palabras, pero solo si la información subléxica de estas lo permite, esto es, si las palabras contienen en su estructura interna una información sobre la que el contexto puede operar. (Sanz Tordesillas, 2019: 194)

Desde esta perspectiva es muy recomendable facilitar esta información subléxica a los alumnos porque les permitirá comprender realmente las razones por las cuales en español es correcta la construcción «celebrar un funeral» pero no lo es «celebrar una autopsia».

10 Es importante tener en cuenta que estos procesos no funcionan en las mismas condiciones si nos comunicamos en una L1 que en una L2. Desde esta evidencia «la necesidad de procesar e interpretar estímulos producidos en una L2 supone una carga cognitiva especial, y que por ello la cantidad de datos que un aprendiente puede procesar (y la velocidad y eficacia con la que puede hacerlo) es mucho más reducida en la L2 que en la L1» (Amenós, Ahern y Escandell, 2019: 27). 
Después de haber señalado algunos conceptos clave en el plano teórico de nuestra disciplina, a continuación, vamos a plantear de qué manera se puede filtrar este conocimiento en una explicación práctica en el aula que permita que el alumno interiorice las combinaciones correctas que se pueden producir con el verbo celebrar acompañado de sustantivos relacionados con la muerte y, al mismo tiempo, facilitarle estrategias para que pueda identificar las erróneas.

\section{Propuesta de una explicación pedagógica y presentación de actividades}

En este punto, lo primero que debe hacerse explícito a los alumnos son las implicaciones que tiene este bloque léxico, puesto que se encuentra ante una combinación léxica formada por un verbo. Tiene que saber que los verbos «seleccionan sus argumentos restringiendo el conjunto de piezas léxicas que pueden denotar de acuerdo con sus rasgos semánticos, por lo que las unidades seleccionadas no suelen ser piezas aisladas, sino clases léxicas» (Jiménez Calderón y Rufat, 2019: 136) Por tanto, como se justificará a continuación, el verbo celebrar puede poseer como argumentos algunos sustantivos como funeral, sepelio, misa o entierro; sin embargo, este verbo no se podrá completar con otros sustantivos como autopsia, levantamiento de cadáver, epitafio o tanatorio. Para que los alumnos puedan utilizar adecuadamente este verbo deberemos trabajar con ellos los rasgos semánticos que posee celebrar ${ }^{11}$.

A continuación, vamos a partir de la definición propuesta por el diccionario de la RAE (DRAE) para iniciar nuestra propuesta explicativa y didactizada dentro del aula ELE. En primer lugar, reproducimos la definición que de la unidad léxica celebrar nos encontramos en el diccionario:

(Del lat. Celebrare. 1. tr. Ensalzar públicamente a un ser sagrado o un hecho solemne, religioso o profano, dedicando uno o más días a su recuerdo. Celebran la Pascua. 2. Tr. Realizar un acto formal con las solemnidades que este requiere. Celebran la boda el estreno y las elecciones. 3. Tr. Realizar un acto festivo por un acto que lo merece. Celebra su cumpleaños. 4. Tr. Mostrar o sentir alegría o agrado por algo. Celebro tu prudente decisión. Celebraron sus simpáticas palabras. 5. Alabar o elogiar a algo o a alguien. La crítica celebró su primera boda. 6. Tr. Dicho de un ministro de la Iglesia: Decir misa. U.t.c.intr. El párroco viene a celebrar a la aldea todos los domingos. 7. Tr. Dicho de un ministro de la Iglesia: Llevar a cabo un acto litúrgico. ${ }^{12}$

Una vez que rescatamos la información léxica que nos facilita el $D R A E$ tenemos la posibilidad de categorizar distintas combinaciones con el verbo celebrar, como se mostrará en la siguiente tabla. Para nuestros estudiantes la combinación más prototípica por su frecuencia de uso, así por la mayor concentración de rasgos prototípicos, es celebrar un cumpleaños. Sin embargo, gracias a la comparativa de rasgos prototípicos entre la categoría más empleada y las demás posibilidades, podrá asimilar

11 El léxico seleccionado tanto en las explicaciones pedagógicas, como en los ejemplos de actividades propuestas, es adecuado a partir de un nivel B2.

12 Diccionario de la lengua española (DRAE). Consúltese en https://dle.rae.es 
los rasgos relacionales y ampliar su disponibilidad léxica con menor esfuerzo y de manera más asumible ${ }^{13}$.

Tabla 1. Caracterización por rasgos de la combinación «celebrar + sustantivo»

\begin{tabular}{|l|c|l|l|l|l|}
\hline & $\begin{array}{c}\text { Acto público } \\
\text { (social/colectivo) }\end{array}$ & Acto religioso & Acto civil & Acto festivo & $\begin{array}{c}\text { Elogiar/ } \\
\text { Alegrarse }\end{array}$ \\
\hline $\begin{array}{l}\text { Celebrar un } \\
\text { funeral }\end{array}$ & + & + & - & - & - \\
\hline $\begin{array}{l}\text { Celebrar un } \\
\text { cumpleaños }\end{array}$ & + & - & $+/-$ & + & + \\
\hline $\begin{array}{l}\text { Celebrar un } \\
\text { bautizo }\end{array}$ & + & + & - & + & + \\
\hline $\begin{array}{l}\text { Celebrar las } \\
\text { elecciones }\end{array}$ & + & - & + & $+/-$ & + \\
\hline $\begin{array}{l}\text { Celebrar la } \\
\text { decisión }\end{array}$ & - & - & - & - & + \\
\hline
\end{tabular}

Si se hace un análisis de la tabla anterior se puede describir que la combinación menos prototípica con el verbo celebrar es «celebrar la decisión»; es por esto por lo que también es la combinación menos frecuente entre los hablantes nativos. Su uso es prácticamente residual por esta diferenciación categorial (sólo posee uno de los rasgos prototípicos de celebrar), por lo que es una combinación claramente periférica. Del mismo modo, su frecuencia de uso es muy restringida por una cuestión asociada al contexto, como hemos explicado, un elemento crítico en el aula de ELE. La combinación «celebrar la decisión» se emplea únicamente en un registro formal (reuniones de empresa, asambleas, discurso político...). Por tanto, los estudiantes tienen escasas posibilidades de adquirirla por contacto con el input. Con esta tabla, los alumnos podrían comprender que «celebrar la decisión» es un sinónimo de «alegrarse por la decisión», restringido a un registro determinado.

Las otras cuatro combinaciones que se presentan en la tabla pertenecen a categorías más prototípicas que la anteriormente explicada. Las cuatro representan actos o eventos sociales/colectivos. Es decir, el alumno puede asumir como uso prototípico de las combinaciones del verbo celebrar que tiene que combinarse con eventos sociales y que, estos pueden ser religiosos o no religiosos/civiles. Este segundo rasgo prototípico permite unir los sustantivos funeral y bautizo (como eventos religiosos) frente a cumpleaños y elecciones (como eventos no religiosos).

Si planteamos a nuestros alumnos la siguiente lista de eventos (funeral, boda civil, Misa del Gallo, aniversario de boda, Domingo de Ramos, despedida de soltero, entierro y acto de graduación), y les pedimos que los categoricen, será sencillo para ellos poder empezar a adquirir combinaciones con el verbo celebrar:

13 En demasiadas ocasiones cuando los estudiantes preguntan al docente por una explicación para comprender por qué un verbo puede combinarse con unos sustantivos y no con otros, el profesor no aporta una explicación basada en los rasgos categoriales y/o subléxicos, sino que sigue pretendiendo que simplemente se memorice (o aporta otro tipo de explicaciones de tipo «intuitivo» que sólo un hablante nativo puede asimilar). Esta actuación pedagógica debe evitarse siempre que sea posible porque en la mayoría de los casos no resulta satisfactoria. 
Tabla 2. Ejemplo de tabla para realizar la actividad propuesta

\begin{tabular}{|l|l|}
\cline { 2 - 2 } \multicolumn{2}{c|}{ CELEBRAR +} \\
\hline ACTO RELIGIOSO & ACTO NO RELIGIOSO \\
\hline & \\
\hline & \\
\hline & \\
\hline & \\
\hline
\end{tabular}

Si tomamos ahora las dos combinaciones de la tabla asociadas a actos religiosos, es decir, «celebrar un funeral» $\mathrm{y}$ «celebrar un bautizo» se puede comprobar que poseen en común la mayoría de los rasgos prototípicos, es decir, son actos religiosos (no civiles) y sociales, la diferenciación en el rasgo prototípico se encuentra en si son festivos o no lo son. Es decir, emocionalmente un bautizo produce alegría, mientras que un funeral es un acto que produce un profundo pesar. De esta manera se rompe la imagen prototípica de «festejo» asociada al verbo celebrar. Sin embargo, todos cumplen el rasgo de «evento».

Por tanto, al tratarse de eventos, si realizamos una actividad asociada a bloques léxicos con términos del campo léxico funerario, los alumnos podrán comprender fácilmente con qué verbos se combinan los diversos bloques. A continuación, presentamos una propuesta de actividad mediante bloques léxicos, basándonos en este prototipo de «evento» asociado al verbo celebrar. Dividimos la actividad en dos partes. En primer lugar, se pide al alumno que indique cuál de los siguientes bloques léxicos se puede combinar con el verbo celebrar. En segundo lugar, para aquellos bloques no combinables con celebrar, se le pide que aporten tres verbos diferentes con los que sí podrían combinarse. Para aportar estos verbos los alumnos deben reflexionar previamente sobre la información subléxica de los sustantivos propuestos con la guía del profesor. Veamos un ejemplo:

Tabla 3. Ejemplo de tabla para realizar la actividad (actividad ya finalizada)

\begin{tabular}{|c|c|c|c|c|c|c|c|}
\hline Deceso & Suceder & Ataúd & Comprar & Funeral & Celebrar & Tanatorio & Asistir \\
\hline Fallecimiento & Ocurrir & Urna & Tener & Responso & & Cementerio & Acudir \\
\hline Óbito & Producirse & Sarcófago & Ver & Sepelio & & Columbario & Visitar \\
\hline
\end{tabular}


La tabla que se acaba de presentar aparece ya completa (tal y como podrían resolverla los alumnos). El primer bloque léxico que se les presenta es el constituido por los sustantivos deceso, fallecimiento y óbito. Los alumnos deben aprender que se trata de sinónimos del sustantivo muerte, asociados a contextos médicos o formales. Por tanto, pueden combinarlos con verbos similares al sustantivo que tienen como referencia, que necesita combinarse con verbos que indiquen la realización de un suceso puntual. El segundo bloque léxico está formado por objetos contenedores de cadáveres o de sus cenizas. Por tanto, estos sustantivos pueden combinarse con verbos transitivos que usualmente se emplean con objetos (verbos que indican posesión, percepción sensorial...). El tercer bloque léxico es el único que sí permite la combinación con celebrar, porque funeral, responso y sepelio son eventos religiosos asociados a los ritos funerarios. Por último, el cuarto bloque léxico está formado por espacios, lugares públicos, asociados a los ritos funerarios. Por tanto, sustantivos como columbario, cementerio o tanatorio, al tratarse de espacios, pueden completarse con verbos como visitar, acudir o asistir. Por tanto, mediante estas cajas léxicas los alumnos podrían realizar combinaciones léxicas asociadas a la categorización de los términos presentados.

A continuación, vamos a seguir trabajando con la tabla anterior para que los alumnos contextualicen las palabras que han categorizado. Se les presentan seis enunciados ${ }^{14}$, y se les da una pareja de opciones para completar. Por tanto, ahora tendrán que asimilar los rasgos diferenciales de los bloques.

1. Fui al .............. (tanatorio/columbario) para dar un abrazo a Cristina.

2. En el imperio egipcio colocaban a las momias en ............ (ataúdes/sarcófagos).

3. Mi vecina todavía tiene las cenizas de su padre en una .......... (urna/ataúd) en el salón.

4. «Es tan frío que he llegado a contestar al ............ (responso/funeral) en nombre de los ausentes» (Diario de Castilla y León, 18/5/2020)

5. Según el parte médico, el .............. (fallecimiento/óbito) se produjo a las cinco y media de la tarde.

6. Las cenizas de nuestro padre están en el la Almudena. (columbario/cementerio) de

Una de las técnicas más recurrentes por su efectividad en el aprendizaje del léxico es la búsqueda de asociación entre una palabra nueva para nuestros alumnos con otra/s que ya conocen. Fijémonos en el siguiente ejemplo. En la primera columna se presenta un conjunto de palabras que ya han adquirido; en la segunda, palabras no conocidas, pero que poseen un determinado nivel de sinonimia con las de la primera columna. Para resolver la actividad es necesario que unan las columnas, pero también deben anotar cuál es la diferencia entre los conceptos, para interiorizar sus diferencias.

\footnotetext{
14 Estos enunciados se presentan como un mero ejemplo para comprender la propuesta. El docente puede crear los que sean más pertinentes en sus respectivos grupos.
} 
RAMO DE FLORES

MISA

CATEDRAL

PENA

ENTERRAMIENTO
CAPILLA

SEPULCRO

CORONA DE FLORES

SEPELIO

PÉSAME

Como última actividad, se debe incluir una actividad de carácter productivo. Para ello, se presenta a los alumnos una actividad orientada a la acción en la que los alumnos ocupen el roll de periodistas. Para ello, se les pide que investiguen sobre los ritos funerarios en un país de habla hispana (sería óptimo que trabajaran por parejas y que cada pareja eligiera un país diferente). Esta investigación les permitirá mejorar su competencia sociocultural. Una vez realizado el trabajo de documentación, los alumnos redactarán de manera cooperativa un reportaje sobre lo que han descubierto y presentarán de manera oral a todo el grupo de clase la información que han encontrado. Por tanto, esta actividad grupal permite la inclusión de las cuatro destrezas y de contenidos lingüísticos y socioculturales también de manera integrada. Consideramos esta última actividad realmente completa y muy adecuada para cerrar esta propuesta.

\section{Conclusiones}

A lo largo del presente artículo se han podido alcanzar algunas consideraciones importantes. La primera de ellas es la necesaria inclusión en las programaciones de ELE de campos semánticos transversales, que no se suelen incluir en las programaciones de manera habitual. Un ejemplo evidente es el léxico asociado a la muerte y a sus ritos, que hemos visto de interés por su importancia sociocultural y léxica, así como por su marcada originalidad, al no reflejarse en los materiales hasta ahora publicados.

En segundo lugar, como se lleva trabajando desde hace años y se ha demostrado científicamente, el enfoque cognitivo y léxico es, sin lugar a duda, el que permite el aprendizaje del léxico con mayores garantías. Por esta razón, mediante la propuesta de actividades que se presenta, los alumnos pueden aprender combinaciones léxicas asociadas a la muerte con una metodología orientada desde estos enfoques.

Las actividades ${ }^{15}$ con las que hemos ejemplificado la puesta en práctica de este enfoque, permiten a los docentes ver plasmada la realización en el aula de un planteamiento teórico concreto, así como demostrar el camino que podrían seguir los estudiantes en su proceso de aprendizaje. Estas actividades, además, pueden ser modificadas y adaptadas de manera sencilla a los distintos contextos didácticos. Por

15 Para el diseño de estas actividades me he basado en las pautas que la doctora Regueiro Rodríguez apunta en su artículo «Algunas reflexiones sobre la enseñanza-aprendizaje del léxico» (2015), en el epígrafe «A modo de conclusión, algunas estrategias didácticas generales». En este texto investigadores y docentes pueden encontrar información de gran interés sobre técnicas didácticas aplicadas al aprendizaje del léxico. 
ello, consideramos que este trabajo resulta de interés tanto para investigadores en enseñanza de ELE, como para docentes que necesiten llevar nuevas propuestas al aula.

\section{Referencias bibliográficas}

AMENÓS, J., AHERN, A. y ESCANDELL, Mํㅡㅁ. (2019). Comunicación y cognición en ELE: la perspectiva pragmática. Madrid: Edinumen.

ARRIBAS ESTERAS, N. (2004). Propuesta de fichas y actividades léxicas para un aprendizaje cognitivo de ELE. En: Ma A. CASTILLO CARBALLO, O. CRUZ MOYA y J. P. MORA GUTIÉRREZ (eds.) (2004). Las gramáticas y los diccionarios en la enseñanza del español como segunda lengua: deseo y realidad (pp. 928-936). (Actas del XV Congreso Internacional de ASELE). Sevilla. Universidad de Sevilla y Universidad Pablo de Olavide.

CUENCA, M. J. y HILFERTY, J. (1999). Introducción a la lingüística cognitiva. Barcelona: Ariel.

FERNÁNDEZ MONTORO, D. (2014). Implicaciones culturales del léxico. Tonos digital: Revista de estudios filológicos, Murcia, Universidad de Murcia, 27, 1-36.

GÓMEZ MOLINA, J. R. (2008). Los contenidos léxico-semánticos. En: J. SÁNCHEZ LOBATO e I. SÁNCHEZ GARGALLO (dirs.) (2008). Vademécum para la formación de profesores. Enseñar español como segunda lengua (L2)/lengua extranjera (LE) (pp. 789-810). Madrid. SGEL.

HIGUERAS GARCÍA, M. (2006). Las colocaciones y su enseñanza en la clase de ELE. Madrid: Arco/Libros.

INSTITUTO CERVANTES. Plan Curricular del Instituto Cervantes. [https://cvc.cervantes.es/ensenanza/biblioteca_ele/plan_curricular/]

JIMÉNEZ CALDERÓN, F. y RUFAT, A. (2019). La enseñanza de verbos frecuentes a partir de enfoques léxicos. Verba Hispánica, Ljubljana, Universidad de Ljubljana, XVII, 131-151.

MÉNDEZ LÓPEZ, M. G. (2016). Las emociones en el aprendizaje de una lengua extranjera. RILE. Revista Internacional de Lenguas extranjeras, Tarragona, Universitat Rovira i Virgili, 5, 27-46.

PENADÉS MARTÍNEZ, I. (2004). La enseñanza de la fraseología en el aula de ELE. Carabela, Madrid, SGEL, 56, 51-67.

PÉREZ SERRANO, M. (2017). La enseñanza-aprendizaje del vocabulario en ELE desde los enfoques léxicos. Madrid: Arco/Libros.

REAL ACADEMIA DE LA LENGUA. Diccionario de la Real Academia de la Lengua (DRAE). https://dle.rae.es/

REGUEIRO RODRÍGUEZ, Mạ L. (2015). Algunas reflexiones sobre la enseñanzaaprendizaje del léxico en ELE. Linred. Lingüística en la Red, Alcalá de Henares, Universidad de Alcalá, 13, 1-35.

SÁNCHEZ RUFAT, A. (2016). Apuntes sobre la revalorización del léxico en la teoría lingüística y en la enseñanza de lenguas. Tejuelo, Badajoz, Universidad de Extremadura, 23, 158-200. 
SANZ TORDESILLAS, A. (2019). Información subléxica, argumental y combinatoria de los verbos saber y conocer. Verba Hispánica, Ljubljana, Universidad de Ljubljana, XVII, 193-208.

TOSCANO-FUENTES, C. y FERNÁNDEZ-CORBACHO, A. (2015). Una enseñanza reflexiva: aspectos cognitivos y afectivos que mejoran el rendimiento en el aula de ELE. MarcoELE, Valencia, 21, 6-24. 